\title{
Example of Parallel Computing Based on Message Passing Interface
}

\author{
ChaoJun Yan \\ College of Computer and Information technology, Three Gorges University, Yi Chang, China \\ aerolus@aliyun.com
}

Keywords: Parallel computing; Message passing interface; Integrated optics; Multimode interference couplers

\begin{abstract}
The alternate directions implicit finite difference beam propagation method is used to analyze the two-dimensional symmetrical multimode interference couplers. The positions of the images at the output plane and the length of multimode waveguide are accurately determined numerically. In order to reduce calculation time, the parallel processing of the arithmetic is implemented by the message passing interface and the simulation is accomplished by 8 personal computers.
\end{abstract}

\section{Introduction}

There has been a growing interest in two-dimensional (2D) multimode interference (MMI) couplers recently. To the actual 2D MMI design, the important parameters concerned are the positions of input or output waveguides and the length of multimode waveguide. JIANG Xiao-qing[1]、Ma Huilian[2]、Yiling Sun[3] have analyzed 2D MMI couplers with guided-mode propagation analysis(MPA) method which is approximate to some extent. In some cases, the modal parameters, for example, the mode propagation constants and even the modal field profiles must be determined before the MPA is used, if there are no analytical solutions which are common for 3D waveguide device, these demands for mode parameters will be obstacles for the use of MPA and restrict analysis precision. Rajarajan[4] obtained the modal propagations and spatial field profiles firstly by the finite element method and used least squares boundary residual method to analyze 2D MMI couplers, so this method is pure numerical and accurate except somewhat complication.

The finite difference beam propagation method (FD-BPM) is a widely used important numeric simulation method for the optical waveguide devices. The alternate directions implicit (ADI) scheme is constructed to improve computing efficiency for the three dimensional device [5]. So, we will use 3D ADI FD-BPM to analyze 2D MMI couplers, it is also a sort of pure numeric method and it need not any modal parameters firstly.

Although the FD-BPM is efficient, there still exists new problem. For a difference scheme, the grid counts at a calculation window must be large enough to guarantee the difference precision, for example, we can use a grid mesh of $1600 \times 50$ to simulate a one dimensional(1D) MMI couplers, the reason why these two numbers which represent $\mathrm{x}$ or $\mathrm{y}$ axes respectively are so different is that the modeling target is a 1D device whose imaging property mainly concentrate on one direction ( $x$ or $y$ ). It is not true for a 2D MMI device for which $1600 \times 1600$ grid mesh maybe used for the same calculation window. The main trouble is that the calculation burden for these two devices is very different; the burden for the 2D device is far more than for the 1D one.

Parallel Computing is one of the optional method to save the calculation time, if we have no super parallel computer, we can use personal computers instead, which can switch messages each other through message passing interface (MPI). MPI is a sort of specification, now the release of MPI has been extended to MPI- 2 . MPICH2, which has been bound with $\mathrm{C}++$ 、 $\mathrm{C}$ or FORTRAN and been integrated into MS Visual Studio developing environment (or Linux), is an implementation of MPI-2 from the group at Argonne National Laboratory. So, we will stress the parallel processing of arithmetic, namely, the total burden of simulation is divided and allocated to more than one computers simultaneously. 


\section{Formulas of parallel algorithm}

Consider an optical beam propagating along $\mathrm{z}$ axis in a MMI, in the finite difference method, the transverse window is divided by a mesh grid with spacing of $\Delta x$ and $\Delta y$, suppose $\Delta z$ is longitudinal step, the field at the lattice point of $x=m \Delta x, y=n \Delta y$ and $z=s \Delta z$ is represented by $E_{m, n}^{s}$. As traditional BPM process, the electric field is expressed as $E(x, y, z)=\phi(x, y, z) e^{i k_{r} z}$, where $k_{r}$ is called reference wave vector and the choice of its value must ensure the slowly varying envelope approximation condition, suppose $\lambda$ is wavelength and $k_{0}=2 \pi / \lambda$ is wave vector in vacuum, $k_{r}$ is usually selected as $k_{r}=k_{0} n_{\text {sub }}$, where $n_{\text {sub }}$ is substrate refractive index of device. The scalar wave equation is:

$$
\partial \phi / \partial z=A\left(\partial^{2} \phi / \partial x^{2}+\partial^{2} \phi / \partial y^{2}\right)+B \phi
$$

where $A=i / 2 k_{r}, B=i\left[k_{0}{ }^{2} n^{2}-k_{r}{ }^{2}\right] / 2 k_{r} \mathrm{n}$ is the refractive index, which is spatially varying.

In the ADI approach, each propagation step from $z$ to $z+\Delta z$ is split into two steps: $z \rightarrow z+\Delta z / 2$ and $z+\Delta z / 2->z+\Delta z$. The difference equation of scalar Helmholtz equation of electric field is as eq. (2):

$$
\begin{gathered}
-A \frac{\Delta z}{\Delta x^{2}} \phi_{m+1, n}^{s+1 / 2}+\left(2+2 A \frac{\Delta z}{\Delta x^{2}}-\frac{\Delta z}{2} B_{m, n}^{s+1 / 2}\right) \phi_{m, n}^{s+1 / 2}-A \frac{\Delta z}{\Delta x^{2}} \phi_{m-1, n}^{s+1 / 2}=A \frac{\Delta z}{\Delta y^{2}}\left(\phi_{m, n-1}^{s}+\phi_{m, n+1}^{s}\right)+\left(2-2 A \frac{\Delta z}{\Delta y^{2}}+\frac{\Delta z}{2} B_{m, n}^{s+1 / 2}\right) \phi_{m, n}^{s} \\
m=1,2 \ldots N_{X}-2 \\
-A \frac{\Delta z}{\Delta y^{2}} \phi_{m, n+1}^{s+1}+\left(2+2 A \frac{\Delta z}{\Delta y^{2}}-\frac{\Delta z}{2} B_{m, n}^{s+1 / 2}\right) \phi_{m, n}^{s+1}-A \frac{\Delta z}{\Delta y^{2}} \phi_{m, n-1}^{s+1}=A \frac{\Delta z}{\Delta x^{2}}\left(\phi_{m-1, n}^{s+1 / 2}+\phi_{m+1, n}^{s+1 / 2}\right)+\left(2-2 A \frac{\Delta z}{\Delta x^{2}}+\frac{\Delta z}{2} B_{m, n}^{s+1 / 2}\right) \phi_{m, n}^{s+1 / 2} \\
n=1,2 \ldots N_{Y}-2
\end{gathered}
$$

Consider equation (2a), for a certain integer $\mathrm{n}$, the range of integer $m$ is from 1 to $N_{X}-2$, so (2a) offers $N_{X}-2$ equations, which, if added two equations educed from boundary condition, will construct a tri-diagonal matrix equation, when this matrix equation is solved, the fields, at the lattice points which are in the line flagged by integer $n$ and in the plane of $z+\Delta z / 2$, are obtained from the fields in the plane of $z$. The range of $n$ is from 0 to $N_{Y}-1$, so if all $N_{Y}$ matrix equations are solved, the fields at the lattice points in the plane of $z+\Delta z / 2$ will be got from the fields in the plane of $z$. The eq. (2b) is similar to (2a), the difference is the rank of the matrix is $N_{Y}$ and the number of matrix equations needed to be solved is $N_{X}$, the fields in the plane of $z+\Delta z$ are obtained from the fields in the plane of $z+\Delta z / 2$.

For the 3D ADI FD-BPM, the paralleling of arithmetic is directly. Let's reconsider (2a), the matrix equation, which corresponds to certain integer ${ }^{n}$ concerned, is constructed by the field at the lattice points in the lines flagged by integer $n-1 、 n$ and $n+1$ on the plane of $z$, the similar matrix equations will be solved $N_{Y}$ times, this property is used for the paralleling of program. According to the MPI terms, the unit which takes part in the paralleling program is called a processor or a thread, for our case, suppose the number of threads which will share the task of solving $N_{Y}$ matrix equations is $N$, the thread with the serial number $p$ will be responsible for the solving of $N_{Y} / N$ matrix equations whose corresponding line flags are from $n=p \times N_{Y} / N$ to $(p+1) \times N_{Y} / N-1$. When constructing a matrix equation, every thread will switch a few messages with adjoining threads only, after that; all ${ }^{N}$ threads will begin operation simultaneously. The similar process is hold for the equation (2b).The threads for the (2a) are called ${ }^{x}$-threads, and the threads for the (2b) are called $y$-threads, when completing the calculation of (2b), ${ }^{y}$ - threads will switch messages with ${ }^{x}$-threads in order to take the next step of BPM, but the quantity of switching, whether from ${ }^{x}$ to ${ }^{y}$ threads or from ${ }^{y}$ to ${ }^{x}$ threads, is much and time-consuming because the memory every thread holds to construct the matrix equations must now be updated totally. 
In the actual implementing, for the convenience of program, every computer offers two threads, one is $x$-thread and the other is $y$-thread, the serial numbers of all $x$-threads are even, the odd number for $y$-threads.

\section{Application of 2D-MMI couplers}

Consider an example of 2D MMI couplers [6][7][8] whose transverse extension of multimode waveguide area is $W_{X}=W_{Y}=30 \mu m$. The cladding and core refractive index are 1.45 and 3.45 respectively, the wavelength is 1.55 . The coordinate origin locates at the geometrical symmetrical center of the cross section of the waveguide, The simulation window in every transverse direction ( ${ }^{x}$ or ${ }^{y}$ ) covers form ${ }^{-30 \mu m}$ to $30 \mu m$, the number of $x$-threads or $y$-threads is $N=8$, and the grid counts is $N_{X}=N_{Y}=1600$,the longitudinal propagation step is $\Delta z=0.2 \mu m$.

The image is input at the center of the waveguide firstly. The simulation aims at the determination of the equivalent width ${ }^{W_{e q}}$ of the MMI couplers. Fig. 1 (a) shows the field profile at the output plane when the length of the MMI is $310 \mu \mathrm{m}$, from the space between the images, we can obtain that $W_{e q}=30.17 \mu \mathrm{m}$.

According to the MPA theory, if the image is not input at point $\left({ }^{i W_{e q} / M}, j W_{e q} / N\right.$ ), where $i, j$ are integer and the point is expressed with absolute coordinates, the $M \times N$ fold-images with equal intensities can be observed at output plane. Suppose $M=N=5$, the input point $(2.8,2.8)$ apparently satisfies the specified condition, Fig. 1 (b) shows the $5 \times 5$ fold-images at output plane when the MMI length is $994^{\mu m}$.the position of one image or of the output waveguide is one of the combinations of the 5 coordinates: $-12.03 、-5.91$ 、 $0.13 、 6.02 、 12.14$.

Finally, the overlapping-images of MMI couplers $[9,10]$ are considered. If image is input at point $\left(i W_{e q} / M, j W_{e q} / N\right)$, the output images will overlap and a new output ratio arises. As an example, let input image is at $\left(W_{e q} / 5,2.8\right)$, the overlapping only exists in the $\mathrm{x}$-direction and the number of images will reduce to 2. Fig. 1 (c) shows the $2 \times 5$ fold-images at output plane when the MMI length is $994^{\mu m}$, the $\mathrm{x}$ coordinates of 10 images are -3.058 and 9.024 , the y coordinates are the same as Fig. 1 (b), the power ratio of two images in the same row is $1: 2.62$ by the calculation of overlap integral [5].

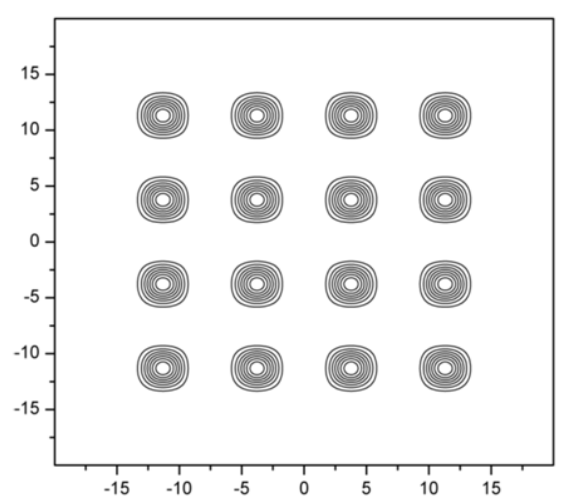

(a)

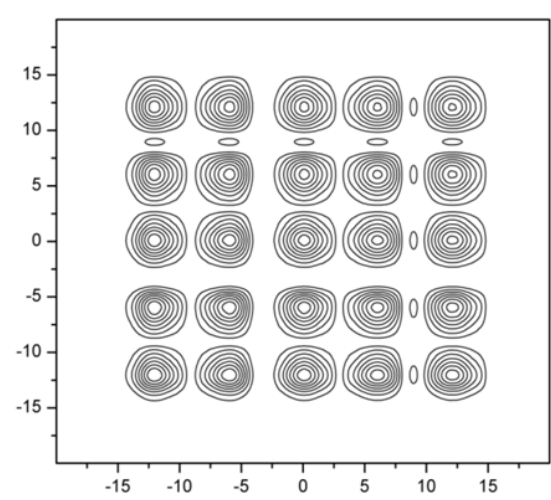

(b) 


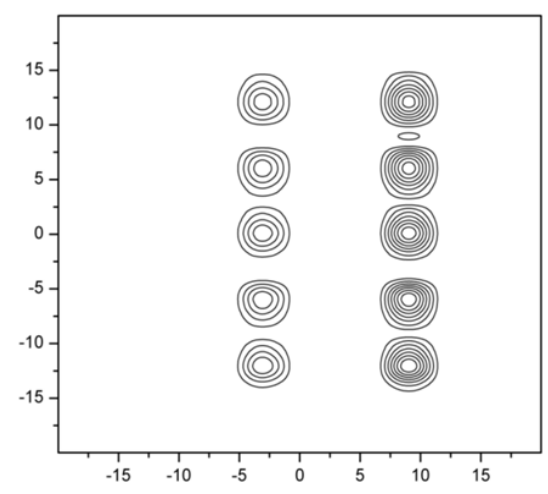

(c)

Fig. 1 Field profile at output plane of MMI couplers (a) $4 \times 4$ images of equal intensity when input field is at center of waveguide; (b) $5 \times 5$ images of equal intensity when not center input; (c) $2 \times 5$ overlapping images without equal intensity

\section{Summary}

This example of MMI couplers shows that the MMI parameters, including the positions of the input or output waveguides and the length of multimode waveguide, are accurately obtained numerically without any modal knowledge ahead, it is indeed as a reference for the actual 2D MMI device.

How about the efficiency of this paralleling program? In our example, $8 \mathrm{x}$-threads and $8 \mathrm{y}$-threads are used, so it is theoretically expected that the simulation time will be decreased to $1 / 8$ of the serial program, in practice, it is only to $1 / 4$ about, the partial reason is that the more messages needed to be transmitted between the $\mathrm{x}$-threads and the $\mathrm{y}$-threads. How to improve the efficiency of the paralleling program is another problem, we after all realize the simulation with desktop computers by the MPI, it shows that the FD-BPM is a powerful method with the convenience and the extensibility of application.

In our example, the configure of desktop machines is : $\mathrm{CPU}$ P4 3.0GHz、512M memory and the network card integrated in mainboard, it takes about 4 minutes per 100 steps of calculation.

\section{References}

[1] Jiang Xiaoqing,Ma Huilian,Mao Huiling,Wang Minghua, "Asymmetrical Self-imaging Effects of MMI Devices with Two-dimensional Confinement", Chinese Journal of Laser,29(No.3),2002:253-256(in Chinese)

[2] Ma Huilian, Jiang Xiaoqing, Wang Minghua, "Self-Imaging Effect Analysis of MMI Devices with Two-Dimensional Confinement”, Acta Optical Sinica,22(No.2),2002:196-200(in Chinese)

[3] Sun Yiling, Jiang Xiaoqing, Tang Yi,Wang Minghua, "Two-Dimensional overlapping-image multimode interference couplers", Chinese Optical Letters,3(No.3),2005:154-155

[4] M. Rajarajan, A. Rahman, T. Wongcharoen, Kenneth T. V. Grattan, “Accurate analysis of MMI device with two-dimensional confinement” , Journal of lightwave technology. 14(9), 1996:2078-2084

[5] Yan Chaojun,Xu Zhenquan,Chen Yixin, "Three dimension finite difference beam propagation method: application to Ti:LiNbO3 directional coupler modeling", Acta Optical Sinica,17(No.6),1997:778-781(in Chinese) 
[6] Dong Xiaye,LiHongqiang,Chen Hongda,LiEnbang,Liu Zhihui,Wei Kejia. 'Design of a $2 \times 2$ MMI Coupler Based on SOI[J]”. Laser \& Optoelectronics Progress, 2012, 49(9): 090602

[7] A. Zahed CHOWDHURY. Performance Study of Silica-on-Silicon Based Multimode Interference (MMI) Optical Coupler[J]. Photonic Sensors, 2014, 4(1): 34-42

[8] Yan Chaojun. Numerical Calculation of Imaging Positions in Multimode Interference Couplers by the Three-Dimensional Beam Propagation Method[J]. Acta Optica Sinica, 2008, 28(1): 110-114

[9] P.P.Sahu, “ATapered structure for compact multimode interfrernce coupler”,IEEE Photonics Tchnology Letters, Vol.20,No.8,April 15,2008,638-640

[10] Homgzhen Wei,Jinzhomg,Zhongli Liu, "Fabrication of $4 \times 4$ tapered MMI coupler with large cross section”, IEEE Photonics Tchnology Letters, vol. 13,No.5,May 2001 